\title{
Oxidative Stress in Intestinal Ischemia-Reperfusion
}

\author{
Guangyao $\mathrm{Li}^{1,2}$, Shuang Wang ${ }^{3 *}$ and Zhe Fan ${ }^{1,2 *}$ \\ 1 Department of General Surgery, The Third People's Hospital of Dalian, Dalian Medical University, Dalian, China, \\ ${ }^{2}$ Department of Central Laboratory, The Third People's Hospital of Dalian, Dalian Medical University, Dalian, China, \\ ${ }^{3}$ Department of Endocrinology, The Second Affiliated Hospital of Dalian Medical University, Dalian, China
}

\section{OPEN ACCESS}

Edited by:

Yanyong Yang,

Second Military Medical

University, China

Reviewed by:

Y-FGu,

Zhejiang University, China

Xinjin Kwok,

The Chinese University of

Hong Kong, China

${ }^{*}$ Correspondence:

Zhe Fan

fanzhe1982@hotmail.com Shuang Wang

wangdalians@hotmail.com

Specialty section:

This article was submitted to

Gastroenterology,

a section of the journal

Frontiers in Medicine

Received: 31 July 2021 Accepted: 16 December 2021

Published: 14 January 2022

Citation:

Li G, Wang S and Fan Z (2022)

Oxidative Stress in Intestinal Ischemia-Reperfusion.

Front. Med. 8:750731.

doi: 10.3389/fmed.2021.750731
Ischemia-reperfusion $(I / R)$ injury is a manifestation of tissue or organ damage that is followed by ischemia and exacerbated by the return of blood flow to a previously damaged tissue or organ. The intestines are one of the most sensitive tissues and organs to I/R injury. Moreover, the adverse consequences of intestinal I/R (II/R) injury are not limited to the intestine itself and can also lead to damage of the distant tissues and organs. The mechanism of $I / R$ is extremely complex and oxidative stress is the key link in the pathogenesis of II/R injury. This study summarizes the roles of oxidative stress and its signaling pathways involved in II/R. The signaling pathways that mitigate II/R injury include the nuclear factor erythroid-related factor 2 (Nrf2)-mediated signaling pathway, Wnt/ $\beta$-catenin pathway, and phosphatidylinositol kinase 3 (PI3K)/Akt pathway; those that aggravate II/R injury include the Janus kinase/signal transducer and activator of transcription (JAK/STAT) pathway, Toll-like receptor (TLR) receptor-mediated signaling pathway, protein kinase C $\beta \|$ (PKC $\beta I) / p 66 s h c$ pathway, and microRNA (miRNA)/p66shc pathway; the effect of miRNA on related pathways and mitochondrial DNA translocation. The aforementioned pathways provide new ideas for further exploring the occurrence and development of II/R and more effective treatments for II/R injury.

Keywords: oxidative stress, intestinal, ischemia-reperfusion, signal pathway, review

\section{INTRODUCTION}

Ischemia-reperfusion ( $\mathrm{I} / \mathrm{R})$ injury is a serious clinical event. In the experimental studies and clinical practice, the ischemia itself does not cause great harm to the organism; instead, the excess reactive oxygen species (ROS) destroy cells as blood flow is restored and this shock directly causes damage to the organism (1). Intestinal $\mathrm{I} / \mathrm{R}$ (II/R) injury is a common and very serious pathophysiological phenomenon (2) often caused by severe trauma, burns, shock, intestinal torsion, mesenteric thromboembolism, and small bowel transplantation (3). II/R damage is a life-threatening pathological event, which is not confined to the intestine and can cause systemic inflammatory response syndrome (SIRS) and multiple organ dysfunction syndrome (MODS), which are closely associated with the high incidence and mortality rates of various diseases (4). The mechanisms involved in II/R injury are quite complex and the response of the intestinal mucosa to I/R can be divided into the following two phases: first, tissue hypoxia and organ damage occur during ischemia; second, large amounts of ROS generated during blood flow reperfusion and reoxygenation trigger an oxidative stress response, which subsequently leads to the destruction of the intestinal mucosal barrier, increased vascular permeability, bacterial translocation, and the release of inflammatory mediators and apoptotic factors (5). 
In the recent years, with more in-depth research, increasing evidence shows that oxidative stress plays a crucial role in the pathogenesis of II/R. This study focuses on the oxidative stress and II/R and a search of the Medical Subject Headings (MeSHs) was carried out using PubMed as follows: oxidative stress, intestinal, gut, and I/R. As oxygen is restored during reperfusion, abundant ROS in damaged cells and tissues can attack almost all the intracellular biomolecules (e.g., cell membranes, organelles, and even DNA) and this oxidative stress disrupts the dynamic homeostasis of the epithelial cells through signal transduction pathways, thereby resulting in the release of large amounts of inflammatory mediators and the induction of apoptosis and exacerbating the damage after reperfusion (6). Mitochondrial DNA (mtDNA) is involved in the oxidative phosphorylation of cells and maintains normal mitochondrial function. After mtDNA is disrupted, ROS production increases and the release of mtDNA into the cytoplasm induces the activation of proinflammatory mediators and proapoptotic factors (7). As the mechanisms of II/R injury have been more extensively investigated, antioxidant pathways and protection of mitochondria against damage have emerged as important means to prevent and treat II/R injury.

\section{OXIDATIVE STRESS}

Oxygen $\left(\mathrm{O}_{2}\right)$ molecules are indispensable for the survival of the body and they provide ATP to the body through the respiratory complex of mitochondria (8). Normal cell metabolism produces ROS, which in small or moderate amounts are beneficial for some physiological processes; however, excessive ROS production during II/R is a significant condition that leads to tissue oxidation and damage to the intestinal epithelial cells (9). ROS originate mainly from the gastrointestinal tract and although intestinal epithelial cells are protected by a mucosal barrier, pathogens can produce inflammatory factors by activating epithelial cells, polymorphonuclear neutrophils, and macrophages and these inflammatory cells release ROS and free radicals in defense against invading pathogens (10). ROS and free radicals are produced to destroy the invading pathogens, but the production of large amounts of ROS adversely affects the homeostasis of the organism and leads to oxidative damage to tissues (11).

Reactive oxygen species include both the free radical compounds and non-radical compounds such as $\mathrm{O}_{2}^{-}, \mathrm{OH}^{-}$, $\mathrm{H}_{2} \mathrm{O}_{2}, \mathrm{O}_{3},{ }^{1} \mathrm{O}_{2}$, and lipid hydrogen peroxide (12). $\mathrm{NO}, \mathrm{NO}_{2}$, $\mathrm{N}_{2} \mathrm{O}_{3}$, and $\mathrm{ONOO}^{-}$are collectively referred to as reactive nitrogen species, which are usually closely associated with ROS (13). These radicals contain unpaired electrons and are, thus, highly chemically reactive toward intracellular proteins, lipids, and even DNA. The activation of ROS can irreversibly damage and deactivate target molecules (14).

Reactive oxygen species can be produced by the mitochondria, endoplasmic reticulum, cell membrane, cytoplasm, nuclei, and peroxidases and can even be produced through extracellular stress (10). Most ROS are produced by mammalian mitochondria; under normal conditions, oxygen molecules are reduced by mitochondrial cytochrome c, thereby forming water and only several oxygen molecules form ROS. Under normal conditions in the body, these free radicals are neutralized by endogenous antioxidant enzymes and, therefore, do not have adverse effects on the organism (15). The mitochondrial respiratory chain regulates ROS production (16). Mitochondrial complexes I and III generate ROS through electron leakage during oxidation (17). Mitochondrial NADPH oxidase and xanthine oxidase catalyze the conversion of $\mathrm{O}_{2}$ to $\mathrm{O}_{2}^{-}$, myeloperoxidase (MPO) catalyzes the conversion of $\mathrm{O}_{2}$ to $\mathrm{OH}^{-}$, and mitochondrial protein kinase $\mathrm{C}$ (PKC) catalyzes the conversion of $\mathrm{O}_{2}$ to $\mathrm{H}_{2} \mathrm{O}_{2}$ (10). Enzymes that catalyze ROSgenerating chemistry in vivo also include lipoxygenases, glucose oxidases, nitric oxide synthases, and cyclooxygenases (18).

Intestinal ischemia can lead to a hypoxic state involving a change in the irreversible conversion of xanthine dehydrogenase (XD) to xanthine oxidase (XO), during which reactive oxygen forms (19). After reperfusion begins and the oxygen supply is restored, electrons from $\mathrm{XO}$ are transferred to molecular oxygen, thus forming significant amounts of oxygen-free radicals such as $\mathrm{O}_{2} \cdot, \mathrm{OH}$, and hydrogen peroxide $\left(\mathrm{H}_{2} \mathrm{O}_{2}\right)$, which injure DNA, cell membranes, and organelles (20). Therefore, II/R injury can decrease villus height, increase cellular infiltration, and aggravate mucosal sloughing in terms of histology. In addition, proinflammatory cytokines are released into the serum including tumor necrosis factor- $\alpha$ (TNF- $\alpha$ ), interleukin 6 (IL-6), interleukin $1 \beta$ (IL-1 $\beta)(21)$.

Although many ROS are produced during oxidative stress, the antioxidants of the body can protect cells and tissues against ROS attack to some extent. Enzymatic antioxidants [Superoxide dismutase (SOD), Glutathione peroxidase (GPX), Glutathione reductase (GSR), Catalase (CAT), and Heme Oxygenase $(\mathrm{HO})]$ and non-enzymatic antioxidants (Glutathione (GSH), Thioredoxin (TRX), and melatonin) play important roles in oxidative stress (22). Exogenous substances, such as vitamin $\mathrm{C}$, vitamin $\mathrm{E}$, carotenoids, mineral ions ( $\mathrm{Mn}$ and $\mathrm{Cu}$ ), and polyphenols, also act as antioxidants $(23,24)$. SOD and CAT play major roles in the antioxidant defense system (25). Therefore, antioxidants scavenge excessive ROS and free radicals during the peroxidation reactions of the body and, thus, may be used to treat II/R damage.

\section{SIGNALING PATHWAYS INVOLVED IN OXIDATIVE STRESS IN II/R}

\section{Pathways to Mitigate Oxidative Stress Nuclear Factor Erythroid-Related Factor 2-Mediated Signaling Pathway}

Nuclear factor erythroid-related factor 2 is a key transcription factor that regulates cellular antioxidative stress and has been found to have roles in the development of many diseases. Under normal conditions, Nrf2 binds the specific repressor protein Keap1 and forms the Nrf2/Keap1 complex, which is localized in the cytoplasm (26). When the body reacts to oxidative stress, Nrf2 dissociates from the Nrf2/Keap1 complex and enters the nucleus, where it maintains the balance of oxidation/antioxidation in the organism by upregulating the expression of antioxidants (HO-1 


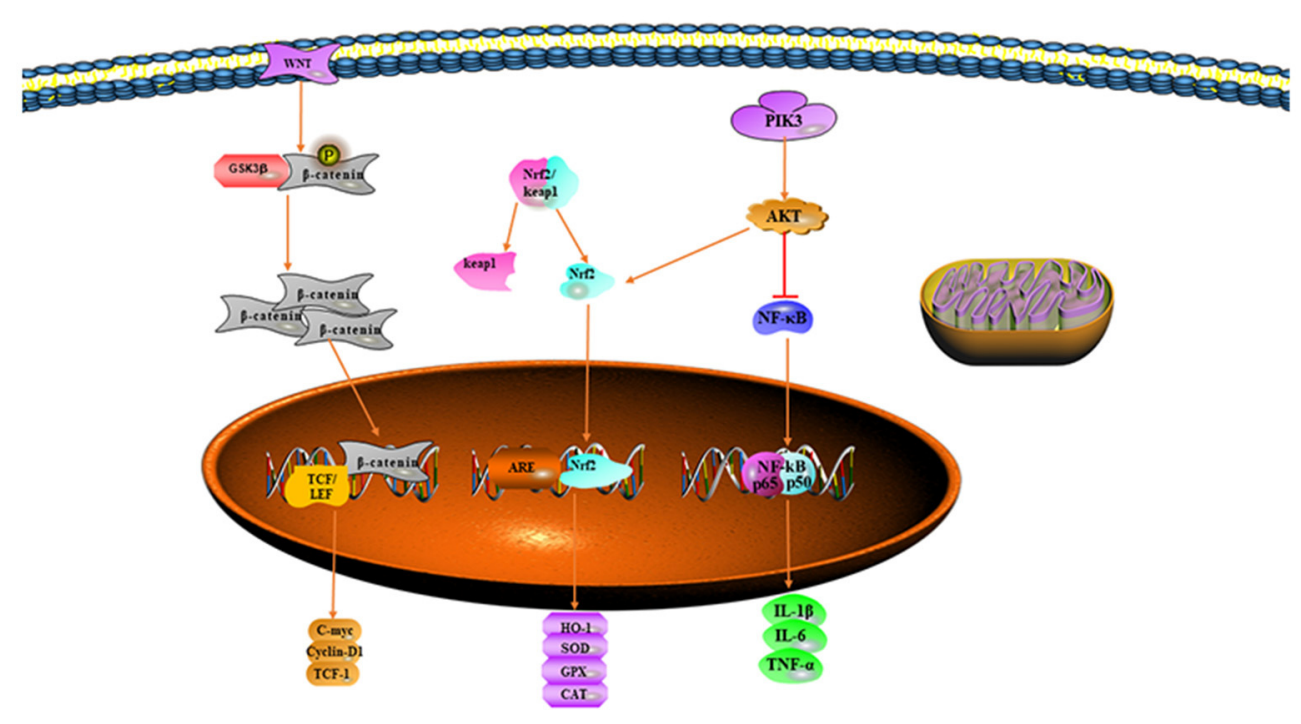

FIGURE 1 | Signaling pathways mitigating oxidative stress.

and SOD) (27). SOD, a specific scavenger of superoxide, catalyzes the decomposition of superoxide anions to $\mathrm{O}^{2-}$ and $\mathrm{H}_{2} \mathrm{O}_{2}$, which, in turn, is disproportionated to $\mathrm{H}_{2} \mathrm{O}$ and $\mathrm{O}_{2}$ through the action of catalase (10). Free heme is extremely lipophilic and inserts into the cell membrane of surrounding cells, consequently activating vascular endothelial cells and leading to an increase in adhesion molecules and inflammatory mediators (28). HO-1 is the enzyme responsible for heme catabolism, catalyzing the degradation of heme and the production of carbon monoxide (CO), bilirubin, and iron (29). Studies have shown that bilirubin has antioxidant effects and is an endogenous antioxidant (30). Although CO is not an antioxidant, it effectively inhibits the release of inflammatory mediators, increases the ratio of the antiapoptotic factor $\mathrm{Bcl}-2$ to the proapoptotic factor Bax, and increases the survival rate in experimental animals (31). HO-1 plays an important role in II/R injury through its antioxidant effects. Because of its antioxidant effect, Nrf2 and its target genes are considered "guardians" of body tissues (32), particularly in intestinal diseases, in which Nrf2 expression improves the intestinal mucosal barrier and decreases the intestinal inflammatory response (33).

Han et al. have found that endogenous lipoxygenin A4 (LXA4) exerts antioxidant effects on II/R-induced oxidative stress by enhancing the Nrf2 signaling pathway and attenuating intestinal mucosal cell damage after I/R (34). Roberta et al. have demonstrated that cashew fruit decreases MPO activity, lipid peroxidation, and ROS by modulating Nrf2/HO-1 signaling, thus restoring antioxidant enzyme activity and significantly decreasing mortality in II/R rats (35). In addition, the use of the anti-inflammatory factor interleukin-1 receptor antagonist (IL-1Ra) not only effectively inhibits the expression of II/R inflammatory factors (IL-1 $\beta$, IL-6, and TNF- $\alpha$ ), but also markedly promotes the expression of $\mathrm{Nrf} 2, \mathrm{HO}-1$, and SOD, thus decreasing intestinal tissue damage (36). Strengthening the Nrf2 pathway and promoting the nuclear translocation of Nrf2 might provide a new therapeutic pathway for treating II/R injury.

\section{Wnt/ $\beta$-Catenin/GSK-3 $\beta$ Signaling Pathway}

Two Wnt signaling pathways are involved in pathophysiological process of the body. The Wnt/ $\beta$-catenin signaling pathway is the classical pathway and the non-classical pathway includes the Wnt/PCP and Wnt/Ca ${ }^{2+}$ pathways; the most widely studied is the $\mathrm{Wnt} / \beta$-catenin signaling pathway (37). The $\mathrm{Wnt} / \beta$-catenin signaling pathway regulates the transcription of many target genes including inflammatory mediators, proapoptotic factors, and ROS associated with II/R injury (38). GSK-3 $\beta$ participates in cell proliferation, apoptosis, and the cell cycle (39). GSK$3 \beta$ inhibits the $\mathrm{Wnt} / \beta$-catenin signaling pathway, and GSK-3 $\beta$ phosphorylates $\beta$-catenin and forms a complex with it in the cytoplasm (40). Wnt signals inactivate GSK-3 $\beta$ and decrease the phosphorylation of $\beta$-catenin. The $\beta$-catenin then enters the nucleus, binds TCF and LEF, and promotes transcription (41).

Previous studies have shown that $\beta$-catenin in the cytoplasm is diminished, thereby decreasing the transcription of other genes under hypoxia (42). Study by Shin et al. has demonstrated that ROS-induced oxidative stress inhibits $\beta$-catenin transcriptional activity (43). When GSK-3 $\beta$ is inhibited by protein kinase B phosphorylation, mitochondrial permeability and mitochondrial membrane potential are decreased, thus attenuating ROS production and protecting mitochondria against damage (39). $\mathrm{Zu}$ et al. have shown that the ginsenoside $\mathrm{Rg} 1$ activates the Wnt/ $\beta$-catenin signaling pathway and decreases II/R-induced inflammatory factors, apoptosis, and ROS (38). Activation of the $\mathrm{Wnt} / \beta$-catenin signaling pathway may become an effective approach to attenuate II/R injury. 


\section{Phosphatidylinositol Kinase 3/Akt Signaling Pathway} Phosphatidylinositol kinase 3 regulates cell proliferation, differentiation, apoptosis, and stress (44). Protein kinase B (Akt), protein kinase $\mathrm{C}(\mathrm{PKC})$, and nuclear factor kappa $\mathrm{B}(\mathrm{NF}-\kappa \mathrm{B})$ recognize $\mathrm{PI} 3 \mathrm{~K}$ and target the activity of the downstream enzymes. When stimulated by extracellular signals, Akt is activated by transmembrane transport and phosphorylation, thereby initiating a series of intracellular reactions that regulate cellular activities (45). The threonine phosphorylation site (Thr308) and serine phosphorylation site (Ser473) on Akt protein are important factors in the activation of Akt (46). Experiments have revealed that activation of the PI3K/Akt signaling pathway protects cells against damage and decreases apoptosis during oxidative stress (47). Inhibition of Akt activation makes cells more sensitive to oxidative stress and more susceptible to damage (48). The PI3K/Akt signaling pathway plays an important role in several antioxidant responses and inflammatory cascade responses (49). Therefore, the PI3K/Akt signaling pathway is an important pathway allowing the body to maintain oxidative homeostasis.

Nuclear factor erythroid-related factor 2 is an important downstream target of the PI3K/Akt pathway (48). The PI3K/Akt pathway acts on $\mathrm{Nrf} 2$ and results in the initiation of the transcription of downstream antioxidant enzymes that protect cells against oxidative stress and inflammatory responses (50). The activation of PI3K/AKT also inhibits the inflammatory response mediated by NF- $\kappa \mathrm{B}$ (51). Chen et al. have found that the ginsenoside $\operatorname{Rg} 1$ attenuates II/R injury by activating the PI3K/Akt pathway, thus inhibiting inflammatory responses and oxidative stress in II/R injury experiments (52). Activation of the PI3K/Akt-mediated antioxidant anti-inflammatory pathway may be a potential therapeutic option to prevent II/R injury (Figure 1).

\section{Pathways That Exacerbate Oxidative Stress \\ Janus Kinase/Signal Transducer and Activator of Transcription Signaling Pathway}

The JAK/STAT signaling pathway is a common pathway in the signaling of many cytokines associated with cell proliferation, differentiation, apoptosis, and inflammation (53). JAKs are a class of non-receptor-type tyrosine kinases. After a cytokine binds its cognate receptor, STATs are activated by phosphorylation of JAKs and the resulting dimer subsequently enters the nucleus and regulates the transcription of target genes (54). Although the JAK/STAT pathway is known to be associated with I/R injury, its effects on I/R injury are quite different. Zhang et al. have suggested that activation of the JAK/STAT signaling pathway by using dexmedetomidine inhibits II/R injury by decreasing caspase-3 and $\mathrm{Bcl}-2 / \mathrm{Bax}$ ratios, thus significantly decreasing apoptosis (53). However, other studies have found that activation of JAK/STAT signaling leads to severe damage to the mucosa after intestinal I/R injury (55). STAT signaling acts on intracellular ROS via the JAK2 pathway and activation of JAK/STAT by oxidative stress may exacerbate II/R injury; in contrast, inhibitors of the JAK/STAT signaling pathway, such as pyruvate, inhibit oxidative stress, decrease neutrophil granulocyte infiltration, regulate microcirculation, and inhibit apoptosis (56). The role of the JAK/STAT signaling pathway in II/R injury requires further research to be clarified.

\section{Toll-Like Receptor-Mediated Signaling Pathways}

Toll-like receptors are a family of receptors expressed in cell membranes and associated with the recognition of pathogenic microorganisms by the immune system, thus allowing for the transmission of extracellular antigen recognition information to the cell (57). As pattern recognition receptors, TLRs recognize not only pathogen-associated molecular patterns (also known as exogenous ligands of TLRs), such as viruses, bacteria, fungi, and other pathogenic microorganisms (57), but also damageassociated molecular patterns (also known as exogenous ligands for TLRs), which are actively secreted or passively released by the organism during stress, as well as molecular patterns (also known as endogenous ligands for TLRs), such as lowdensity lipoproteins and heat shock proteins, apoptotic cells, and nucleic acids (58). During II/R, excessive ROS also stimulates the activation of TLRs and consequently aggravates the inflammatory response (59). Activated TLRs trigger a systemic inflammatory response by binding to the $\mathrm{C}$-terminus of the junction protein MyD88 and then to downstream target genes [mitogen-activated protein kinase (MAPK) and NF- $\mathrm{B}$ ] through the N-terminal end of the MyD88 molecule, thus inducing the release of inflammatory mediators (IL-1 $\beta$, IL-6, and TNF- $\alpha$ ) (60). In most models of intestinal inflammation, TLR4 induces inflammatory responses more commonly than other TLRs. Marwan has shown that cinnamaldehyde pretreatment attenuates oxidative stress by restoring the levels of SOD, GSH, Lactic Dehydrogenase (LDH), and Malondialdehyde (MDA) in I/R-treated intestinal tissues; it also attenuates intestinal injury by inhibiting the expression of p65 and p50 in the NF-кB pathway (61). TLRmediated inflammatory responses and apoptosis clearly have strong adverse effects on cells and tissues in II/R; therefore, targeting TLR pathways for inhibition may be a good choice for treating II/R injury.

\section{Protein Kinase C $\beta$ III/P66shc Pathway}

Protein kinase $\mathrm{C}$ is a multifunctional protein kinase that is normally present in the cytoplasm. When cells are stimulated, PKC translocates from the cytoplasm to the cell membrane in the form of $\mathrm{Ca}^{2+}$, where it plays an important role in signal transduction pathways through phosphorylation (62). Among the various PKC isoforms, PKC $\beta I I$ is activated in II/R injury (63). The bridging protein p66shc is an important regulator of oxidative stress in mitochondria and cells. When oxidative stress occurs, p66shc is phosphorylated and after dephosphorylation by protein serine-threonine phosphatase, it translocates to mitochondria, where it promotes ROS production (64). Phosphorylated p66shc promotes the phosphorylation of FOXO3a, which becomes inactive when phosphorylated, and active FOXO3a upregulates the expression of the antioxidant enzyme SOD (65). In addition, p66shc causes the release of mitochondrial cytochrome c. Cytochrome c entering the cytoplasm leads to the activation of the proapoptotic factors 


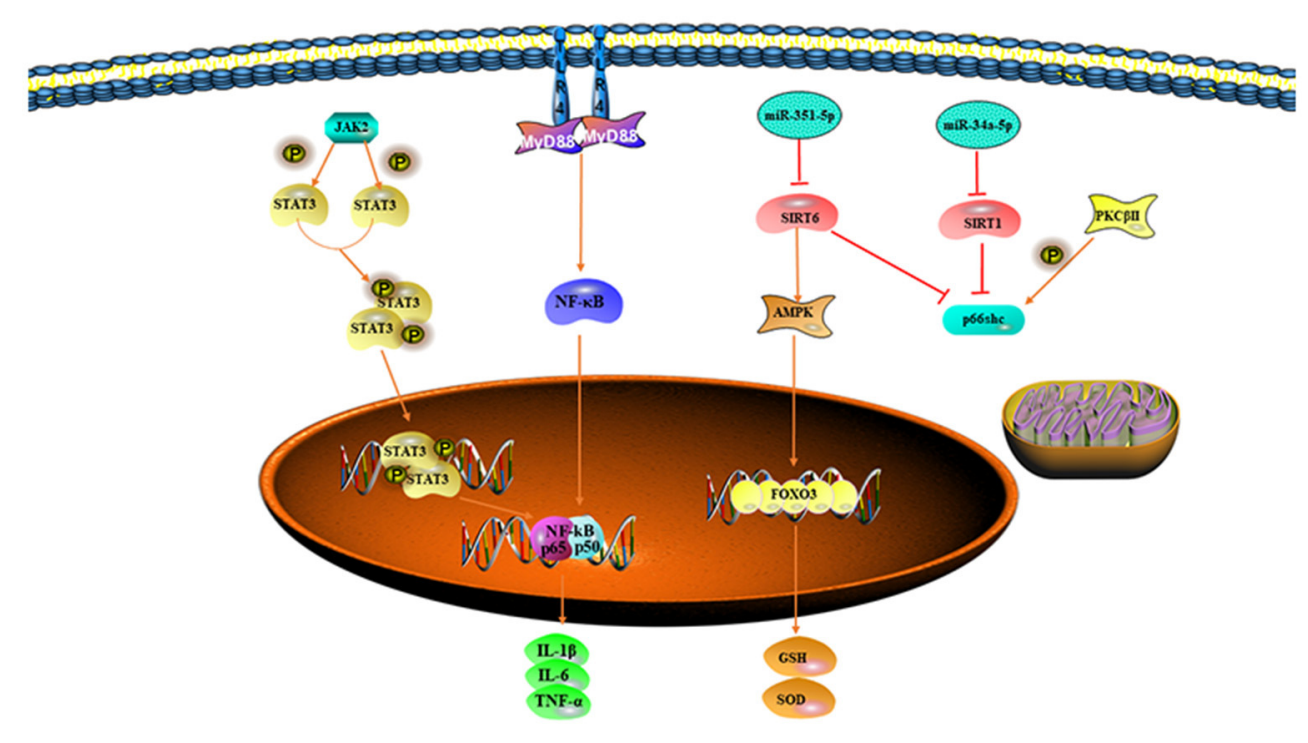

FIGURE 2 | Signal pathways that exacerbate oxidative stress.

caspase-3, 6, and $9(66,67)$. Wang has shown that LY333531, which inhibits the $\mathrm{PKC} \beta \mathrm{II} / \mathrm{p} 66 \mathrm{shc}$ pathway, attenuates damage to the intestines in II/R (68). Therefore, inhibition of the PKC $3 I$ I/p66shc pathway, which not only decreases ROS production but also inhibits the activation of proapoptotic factors, has a significant effect on the treatment of II/R injury.

\section{Effect of microRNA on Mechanisms Involved in II/R Injury}

MicroRNA is a type of highly conserved single-stranded noncoding RNA widely found in plants and animals. It negatively regulates the expression of target genes after transcription through complementary pairing with mRNA (69). In the recent years, numerous studies have found that II/R increases or decreases miRNA expression levels in intestinal tissues and these effects play varying roles in II/R damage depending on the mechanism of action of downstream target genes (70). Numerous studies have shown that miR-23a-5p (71), miR-34a-5p (66), and miR-351-5p (72), isoforms of miRNAs, affect II/R injury to some extent by regulating a series of signal transduction pathways.

Sirtuin1 (SIRT1) is a histone deacetylase whose function depends on nicotinamide adenine dinucleotide. Because of its important targets, SIRT1 has roles in regulating cellular metabolism, senescence, redox homeostasis, and apoptosis (73). SIRT1 mediates oxidative responses by regulating transcription factors of antioxidant genes. It binds the promoter of p66Shc, deacetylates p66Shc, and decreases the transcription and translation of p66shc, thereby regulating ROS levels and inducing apoptosis (74). Owing to the antioxidant effect of SIRT1, numerous studies have linked SIRT1 to I/R injury, implying that SIRT1 plays a key role in II/R-induced oxidative stress (75). Wang et al. have experimentally found that miR-34a-5p inhibits SIRT1mediated signaling pathways, thus leading to the upregulation of p66shc, the reduction of MnSOD and the activation of caspase-3, and exacerbating II/R injury (66).

Mitogen-activated protein kinase 13 is a type of p38 MAPK that links extracellular signals to intracellular signals and regulates processes such as apoptosis, the inflammatory response, differentiation, and senescence (76). SIRT6 is a member of the sirtuin family that is involved in the cellular processes such as inflammatory responses, oxidative stress, and apoptosis (77). SIRT6 decreases apoptosis by binding specific sites in the Bax promoter and it improves the antioxidant capacity of the body by activating FOXO3a, either directly or through activation of Adenosine $5^{\prime}$-monophosphate (AMP)-activated protein kinase (AMPK) (78). Hu et al. have experimentally found that MAPK13 and SIRT6 are the downstream target genes of miR-351-5p. During II/R, miRNA-351-3p is significantly upregulated and MAPK13 and SIRT6 expression are significantly decreased, thus leading to the activation of their downstream inflammatory response, oxidative stress, and apoptotic signaling pathways and increasing the degree of intestinal damage (72) (Figure 2).

\section{MITOCHONDRIAL DNA TRANSLOCATION AGGRAVATES OXIDATIVE STRESS}

Mitochondria, important organelles in eukaryotic cells, are involved in energy supply, apoptosis, maintenance of intracellular ion homeostasis, and the production and removal of ROS. When pathogenic microorganisms infect the host, mitochondria have important roles in organisms by inducing multiple immune responses to clear the infected cells (79). mtDNA, genetic material located within the inner membrane of mitochondria, is involved in oxidative phosphorylation as well as maintaining normal mitochondrial function (80). Oxidative stress is likely to damage mtDNA because of proximity of the 


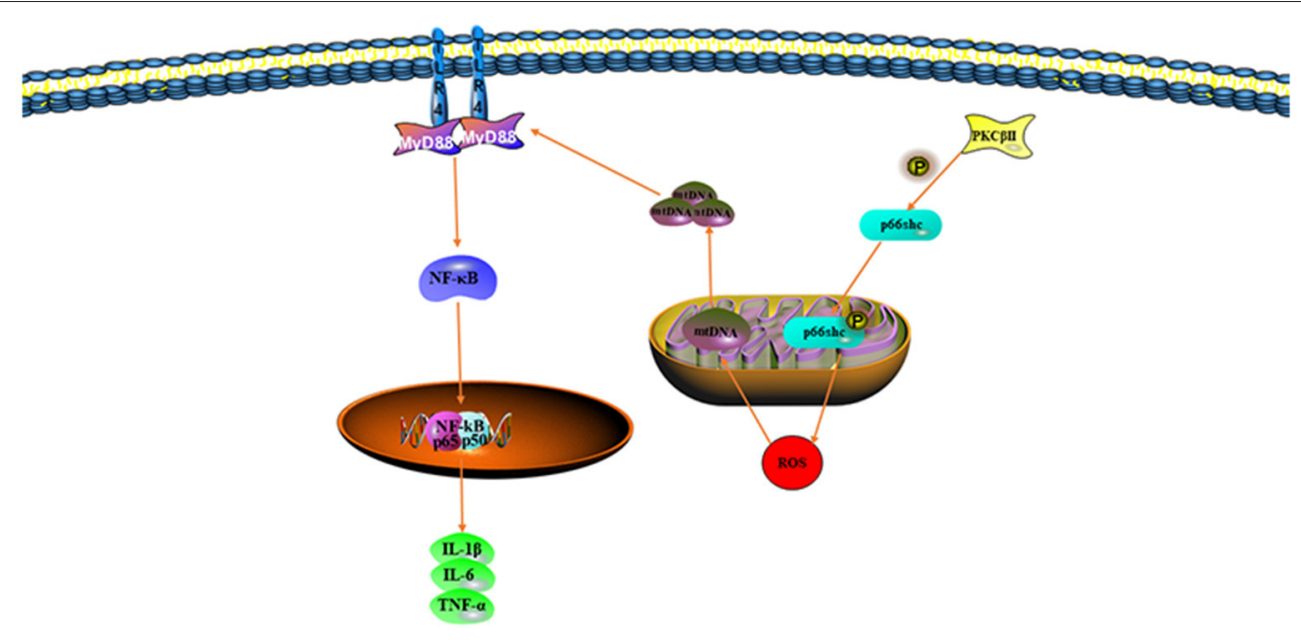

FIGURE 3 | Mitochondrial DNA (mtDNA) translocation aggravates oxidative stress.

mtDNA to the respiratory chain and its lack of protective histones (81). After oxidative stress damage occurs, the continual increase in damaged mtDNA destroys the function of mitochondria. mtDNA copy and gene transcription levels decrease, thereby affecting the formation of the respiratory chain complex and leading to mitochondrial dysfunction. Mitochondrial dysfunction, in turn, promotes massive production of ROS, in a cycle that eventually leads to apoptosis (82).

During II/R injury, the body initiates damage-associated molecular patterns, many of which are derived from mitochondria (83). Normally, mtDNA is present between the inner and outer mitochondrial membranes. However, during II/R, mitochondria are stimulated by oxidative stress and mtDNA fragments are released into the cytoplasm, thereby triggering a series of inflammatory responses (84). Several studies have shown that mtDNA damage results in the release of inflammatory factors (IL-1 $\beta$, IL-6, and TNF- $\alpha$ ) through TLRs and the NOD-like receptor protein 3 (NLRP3) signaling pathway (85). $\mathrm{Hu}$ et al. have found that the mitochondria-targeted antioxidant MitoQ decreases mitochondrial ROS production through activation of the Nrf2 pathway, decreases oxidative stress, and improves mtDNA translocation caused by II/R damage (86) (Figure 3).

\section{CONCLUSION}

Intestinal ischemia-reperfusion injury is a pathological condition involving multiple organs; reperfusion is necessary to maintain

\section{REFERENCES}

1. Wu MY, Yiang GT, Liao WT, TCompr Physiol.sai AP, Cheng YL, Cheng PW, et al. Current mechanistic concepts in ischemia and reperfusion injury. Cell Physiol Biochem. (2018) 46:1650-67. doi: 10.1159/000 489241 homeostasis, but reoxygenation during reperfusion produces great damage to the organism. Excessive oxidative stress can lead to damage to cell membranes, organelles, and even DNA. Therefore, decreasing ROS production and strengthening the antioxidant defense system play important roles in the treatment of II/R injury. However, the mechanism of ROS production and the molecular mechanism of ROS that leads to tissue damage is very complex (Figure 1). With the continual progress of related research, a breakthrough has been achieved in treating excessive ROS and decreasing oxidative stress. Even greater breakthroughs are soon expected in the development, prevention, and treatment of II/R injury.

\section{AUTHOR CONTRIBUTIONS}

GL, SW, and ZF contributed to conception and design of the article. GL wrote the first draft of the manuscript. SW and ZF wrote sections of the manuscript and revised the manuscript. All authors contributed to manuscript revision, read, and approved the submitted version.

\section{FUNDING}

Information retrieval was done by the National Natural Science Foundation of China (81701965) and the Natural Science Foundation of Liaoning Province (2020-BS-187). Physiol. (2016) 7:113-70. doi: 10.1002/cphy.c160006

3. Wen J, Xu B, Sun Y, Lian M, Li Y, Lin Y, et al. Paeoniflorin protects against intestinal ischemia/reperfusion by activating LKB1/AMPK and promoting autophagy. Pharmacol Res. (2019) 146:104308. doi: 10.1016/j.phrs.2019.104308 
4. Tang J, Zhuang S. Histone acetylation and DNA methylation in ischemia/reperfusion injury. Clin Sci. (2019) 133:597609. doi: $10.1042 / C S 20180465$

5. Zhao W, Gan X, Su G, Wanling G, Li S, Hei Z, et al. The interaction between oxidative stress and mast cell activation plays a role in acute lung injuries induced by intestinal ischemia-reperfusion. J Surg Res. (2014) 187:542-52. doi: 10.1016/j.jss.2013.10.033

6. Perez S, Talens-Visconti R, Rius-Perez S, Finamor I, Sastre J. Redox signaling in the gastrointestinal tract. Free Radic Biol Med. (2017) 104:75103. doi: $10.1016 /$ j.freeradbiomed.2016.12.048

7. Mikhed Y, Daiber A, Steven S. Mitochondrial oxidative stress, mitochondrial DNA damage and their role in age-related vascular dysfunction. Int J Mol Sci. (2015) 16:15918-53. doi: 10.3390/ijms160715918

8. Larosa $\mathrm{V}$, Remacle $\mathrm{C}$. Insights into the respiratory chain and oxidative stress. Biosci Rep. (2018) 38:BSR20171492. doi: 10.1042/BSR20171492

9. Peng Z, Ban K, Wawrose RA, Gover AG, Kozar RA. Protection by enteral glutamine is mediated by intestinal epithelial cell peroxisome proliferatoractivated receptor-gamma during intestinal ischemia/reperfusion. Shock. (2015) 43:327-33. doi: 10.1097/SHK.0000000000000297

10. Bhattacharyya A, Chattopadhyay R, Mitra S, Crowe SE. Oxidative stress: an essential factor in the pathogenesis of gastrointestinal mucosal diseases. Physiol Rev. (2014) 94:329-54. doi: 10.1152/physrev.00040.2012

11. Cieslar-Pobuda A, Yue J, Lee HC, Skonieczna M, Wei YH. ROS and oxidative stress in stem cells. Oxid Med Cell Longev. (2017) 2017:5047168. doi: 10.1155/2017/5047168

12. Herman J, Zhang Y, Castranova V, Neal SL. Emerging technologies for optical spectral detection of reactive oxygen species. Anal Bioanal Chem. (2018) 410:6079-95. doi: 10.1007/s00216-018-1233-1

13. Tharmalingam S, Alhasawi A, Appanna VP, Lemire J, Appanna VD. Reactive nitrogen species (RNS)-resistant microbes: adaptation and medical implications. Biol Chem. (2017) 398:1193-208. doi: 10.1515/hsz-2017-0152

14. Juan CA, Perez de la Lastra JM, Plou FJ, Perez-Lebena E. The chemistry of reactive oxygen species (ROS) revisited: outlining their role in biological macromolecules (DNA, lipids and proteins) and induced pathologies. Int $J$ Mol Sci. (2021) 22:4642. doi: 10.3390/ijms22094642

15. Singh A, Kukreti R, Saso L, Kukreti S. Oxidative stress: a key modulator in neurodegenerative diseases. Molecules. (2019) 24:1583. doi: $10.3390 /$ molecules 24081583

16. Poyton RO, Castello PR, Ball KA, Woo DK, Pan N. Mitochondria and hypoxic signaling: a new view. Ann N Y Acad Sci. (2009) 1177:4856. doi: 10.1111/j.1749-6632.2009.05046.x

17. Sarniak A, Lipinska J, Tytman K, Lipinska S. Endogenous mechanisms of reactive oxygen species (ROS) generation. Postepy Hig Med Dosw. (2016) 70:1150-65. doi: 10.5604/17322693.1224259

18. Swindle EJ, Metcalfe DD. The role of reactive oxygen species and nitric oxide in mast cell-dependent inflammatory processes. Immunol Rev. (2007) 217:186-205. doi: 10.1111/j.1600-065X.2007.00513.x

19. Akinrinmade FJ, Akinrinde AS, Soyemi OO, Oyagbemi AA. Antioxidant potential of the methanol extract of parquetina nigrescens mediates protection against intestinal ischemia-reperfusion injury in rats. J Diet Suppl. (2016) 13:420-32. doi: 10.3109/19390211.2015.1103828

20. Prieto-Moure B, Cejalvo-Lapena D, Belda-Antoli M, Padron-Sanz C, Lloris-Cejalvo JM, Lloris-Carsi JM. Combination therapy of allopurinol and dantrolene and its role in the prevention of experimental ischemia reperfusion injury of the small intestine. J Invest Surg. (2021) 34:8007. doi: 10.1080/08941939.2019.1696904

21. Wang Y, Wen J, Almoiliqy M, Wang Y, Liu Z, Yang X, et al. Sesamin protects against and ameliorates rat intestinal ischemia/reperfusion injury with involvement of activating Nrf2/HO-1/NQO1 signaling pathway. Oxid Med Cell Longev. (2021) 2021:5147069. doi: 10.1155/2021/5147069

22. Neha K, Haider MR, Pathak A, Yar MS. Medicinal prospects of antioxidants: a review. Euro J Med Chem. (2019) 178:687704. doi: 10.1016/j.ejmech.2019.06.010

23. Miyazawa $T$, Burdeos GC, Itaya $M$, Nakagawa $K$, Miyazawa $T$. Vitamin E: Regulatory redox interactions. IUBMB Life. (2019) 71:430-41. doi: 10.1002/iub.2008
24. Ji M, Gong X, Li X, Wang C, Li M. Advanced research on the antioxidant activity and mechanism of polyphenols from hippophae species-a review. Molecules. (2020) 25:917. doi: 10.3390/molecules25040917

25. Palma FR, He C, Danes JM, Paviani V, Coelho DR, Gantner BN, et al. Mitochondrial superoxide dismutase: what the established, the intriguing, and the novel reveal about a key cellular redox switch. Antioxid Redox Signal. (2020) 32:701-14. doi: 10.1089/ars.2019.7962

26. Yang B, Bai Y, Yin C, Qian H, Xing G, Wang S, et al. Activation of autophagic flux and the Nrf2/ARE signaling pathway by hydrogen sulfide protects against acrylonitrile-induced neurotoxicity in primary rat astrocytes. Arch Toxicol. (2018) 92:2093-108. doi: 10.1007/s00204-018-2208-x

27. Tebay LE, Robertson H, Durant ST, Vitale SR, Penning TM, Dinkova-Kostova AT, et al. Mechanisms of activation of the transcription factor Nrf2 by redox stressors, nutrient cues, and energy status and the pathways through which it attenuates degenerative disease. Free Radic Biol Med. (2015) 88 (Pt. B):108-46. doi: 10.1016/j.freeradbiomed.2015.06.021

28. Pradhan P, Vijayan V, Gueler F, Immenschuh S. Interplay of heme with macrophages in homeostasis and inflammation. Int J Mol Sci. (2020) 21:740. doi: $10.3390 /$ ijms 21030740

29. Nadatani Y, Watanabe T, Shimada S, Otani K, Tanigawa T, Fujiwara Y. Microbiome and intestinal ischemia/reperfusion injury. J Clin Biochem Nutr. (2018) 63:26-32. doi: 10.3164/jcbn.17-137

30. Maruhashi T, Kihara Y, Higashi Y. Bilirubin and endothelial function. $J$ Atheroscler Thromb. (2019) 26:688-96. doi: 10.5551/jat.RV17035

31. Takagi T, Naito Y, Higashimura Y, Uchiyama K, Okayama T, Mizushima K, et al. Rectal administration of carbon monoxide inhibits the development of intestinal inflammation and promotes intestinal wound healing via the activation of the Rho-kinase pathway in rats. Nitric Oxide. (2021) 107:1930. doi: 10.1016/j.niox.2020.12.006

32. Lau WL, Liu SM, Pahlevan S, Yuan J, Khazaeli M, Ni Z, et al. Role of Nrf2 dysfunction in uremia-associated intestinal inflammation and epithelial barrier disruption. Dig Dis Sci. (2015) 60:1215-22. doi: 10.1007/s10620-014-3428-4

33. Wen Z, Liu W, Li X, Chen W, Liu Z, Wen J, et al. A Protective Role of the NRF2-Keap1 Pathway in Maintaining Intestinal Barrier Function. Oxidative medicine and cellular longevity. (2019) 2019:1759149. doi: 10.1155/2019/1759149

34. Han X, Yao W, Liu Z, Li H, Zhang ZJ, Hei Z, et al. Lipoxin A4 preconditioning attenuates intestinal ischemia reperfusion injury through Keap1/Nrf2 pathway in a lipoxin A4 receptor independent manner. Oxid Med Cell Longev. (2016) 2016:9303606. doi: 10.1155/2016/9303606

35. Fusco R, Cordaro M, Siracusa R, Peritore AF, Gugliandolo E, Genovese T, et al. Consumption of anacardium occidentale L. (cashew nuts) inhibits oxidative stress through modulation of the Nrf2/HO-1 and NF-kB pathways. Molecules. (2020) 25:4426. doi: 10.3390/molecules 25194426

36. Jin $\mathrm{C}, \mathrm{Fu} \mathrm{WL}$, Zhang DD, Xing WW, Xia WR, Wei Z, et al. The protective role of IL-1Ra on intestinal ischemia reperfusion injury by antioxidative stress via Nrf2/HO-1 pathway in rat. Biomed J. (2019) 42:3645. doi: 10.1016/j.bj.2018.11.001

37. Katoh M. Multilayered prevention and treatment of chronic inflammation, organ fibrosis and cancer associated with canonical WNT/betacatenin signaling activation (Review). Int J Mol Med. (2018) 42:713-25. doi: 10.3892/ijmm.2018.3689

38. Zu G, Guo J, Che N, Zhou T, Zhang X, Wang G, et al. Protective effects of ginsenoside $\mathrm{Rg} 1$ on intestinal ischemia/reperfusion injury-induced oxidative stress and apoptosis via activation of the Wnt/beta-catenin pathway. Sci Rep. (2016) 6:38480. doi: 10.1038/srep38480

39. Yang K, Chen Z, Gao J, Shi W, Li L, Jiang S, et al. The key roles of GSK-3beta in regulating mitochondrial activity. Cell Physiol Biochem. (2017) 44:1445-59. doi: 10.1159/000485580

40. Doble BW, Woodgett JR. GSK-3: tricks of the trade for a multi-tasking kinase. J Cell Sci. (2003) 116 (Pt. 7):1175-86. doi: 10.1242/jcs.00384

41. Abd El-Fadeal NM, Nafie MS, M KE-K, El-Mistekawy A, Mohammad HMF, Elbahaie AM, et al. Antitumor activity of nitazoxanide against colon cancers: molecular docking and experimental studies based on Wnt/beta-catenin signaling inhibition. Int J Mol Sci. (2021) 22:5213. doi: 10.3390/ijms22105213 
42. Kaidi A, Williams AC, Paraskeva C. Interaction between beta-catenin and HIF-1 promotes cellular adaptation to hypoxia. Nat Cell Biol. (2007) 9:2107. doi: $10.1038 /$ ncb1534

43. Shin SY, Chin BR, Lee YH, Kim JH. Involvement of glycogen synthase kinase-3beta in hydrogen peroxide-induced suppression of Tcf/Lef-dependent transcriptional activity. Cell Signal. (2006) 18:601-7. doi: 10.1016/j.cellsig.2005.06.001

44. Xue X, Wang L, Meng X, Jiao J, Dang N. Regulator of G protein signaling 4 inhibits human melanoma cells proliferation and invasion through the PI3K/AKT signaling pathway. Oncotarget. (2017) 8:7853044. doi: 10.18632/oncotarget.20825

45. Huang L, Cao J, Cao L, Gao L, Yang Y, Xu L. Puerarin induces cell apoptosis in human chondrosarcoma cell line SW1353 via inhibition of the PI3K/Akt signaling pathway. Oncol Lett. (2017) 14:5585-90. doi: 10.3892/ol.2017.6901

46. Manning BD, Toker A. AKT/PKB signaling: navigating the network. Cell. (2017) 169:381-405. doi: 10.1016/j.cell.2017.04.001

47. Duarte AI, Santos P, Oliveira CR, Santos MS, Rego AC. Insulin neuroprotection against oxidative stress is mediated by Akt and GSK-3beta signaling pathways and changes in protein expression. Biochim Biophys Acta. (2008) 1783:994-1002. doi: 10.1016/j.bbamcr.2008.02.016

48. Moniruzzaman M, Ghosal I, Das D, Chakraborty SB. Melatonin ameliorates $\mathrm{H} 2 \mathrm{O} 2$-induced oxidative stress through modulation of Erk/Akt/NFkB pathway. Biol Res. (2018) 51:17. doi: 10.1186/s40659-018-0168-5

49. Yu M, Qi B, Xiaoxiang W, Xu J, Liu X. Baicalein increases cisplatin sensitivity of A549 lung adenocarcinoma cells via PI3K/Akt/NF-kappaB pathway. Biomed Pharmacother. (2017) 90:677-85. doi: 10.1016/j.biopha.2017.04.001

50. Zhang R, Chae S, Lee JH, Hyun JW. The cytoprotective effect of butin against oxidative stress is mediated by the up-regulation of manganese superoxide dismutase expression through a PI3K/Akt/Nrf2-dependent pathway. J Cell Biochem. (2012) 113:1987-97. doi: 10.1002/jcb.24068

51. Kai-lan W, Si Z. Pretreatment with erythropoietin attenuates intestinal ischemia reperfusion injury by further promoting PI3K/Akt signaling activation. Transplant Proc. (2015) 47:163945. doi: 10.1016/j.transproceed.2015.02.023

52. Chen S, Li X, Wang Y, Mu P, Chen C, Huang P, et al. Ginsenoside Rb1 attenuates intestinal ischemia/reperfusioninduced inflammation and oxidative stress via activation of the PI3K/Akt/Nrf2 signaling pathway. Mol Med Rep. (2019) 19:3633-41. doi: 10.3892/mmr.2019.10018

53. Zhang X, Zhou J, Hu Q, Liu Z, Chen Q, Wang W, et al. The role of janus kinase/signal transducer and activator of transcription signalling on preventing intestinal ischemia/reperfusion injury with dexmedetomidine. $J$ Nanosci Nanotechnol. (2020) 20:3295-302. doi: 10.1166/jnn.2020.16416

54. Xin P, Xu X, Deng C, Liu S, Wang Y, Zhou X, et al. The role of JAK/STAT signaling pathway and its inhibitors in diseases. Int Immunopharmacol. (2020) 80:106210. doi: 10.1016/j.intimp.2020.106210

55. Wen SH, Li Y, Li C, Xia ZQ, Liu WF, Zhang XY, et al. Ischemic postconditioning during reperfusion attenuates intestinal injury and mucosal cell apoptosis by inhibiting JAK/STAT signaling activation. Shock. (2012) 38:411-9. doi: 10.1097/SHK.0b013e3182662266

56. Zhang JJ, Deng JT, Shen HQ, Jiang LL, He QW, Zhan J, et al. Pyruvate protects against intestinal injury by inhibiting the JAK/STAT signaling pathway in rats with hemorrhagic shock. J Surg Res. (2020) 248:98108. doi: 10.1016/j.jss.2019.11.012

57. Satoh T, Akira S. Toll-Like receptor signaling and its inducible proteins. Microbiol Spectr. (2016) 4:1-7. doi: 10.1128/microbiolspec.MCHD-0040-2016

58. De Nardo D. Toll-like receptors: activation, signalling and transcriptional modulation. Cytokine. (2015) 74:181-9. doi: 10.1016/j.cyto.2015.02.025

59. Fink MP, Macias CA, Xiao J, Tyurina YY, Jiang J, Belikova N, et al. Hemigramicidin-TEMPO conjugates: novel mitochondriatargeted anti-oxidants. Biochem Pharmacol. (2007) 74:8019. doi: 10.1016/j.bcp.2007.05.019

60. Ali M, Yang F, Jansen JA, Walboomers XF. Lipoxin suppresses inflammation via the TLR4/MyD88/NF-kappaB pathway in periodontal ligament cells. Oral Dis. (2020) 26:429-38. doi: 10.1111/odi.13250

61. Almoiliqy M, Wen J, Xu B, Sun YC, Lian MQ, Li YL, et al. Cinnamaldehyde protects against rat intestinal ischemia/reperfusion injuries by synergistic inhibition of NF-kappaB and p53. Acta Pharmacol Sin. (2020) 41:120822. doi: 10.1038/s41401-020-0359-9
62. Garcia G, Mendez-Resendiz KA, Oviedo N, Murbartian J. PKC- and PKA-dependent phosphorylation modulates TREK-1 function in naive and neuropathic rats. J Neurochem. (2021) 157:2039-54. doi: 10.1111/jnc.15204

63. Chen Z, Wang G, Zhai X, Hu Y, Gao D, Ma L, et al. Selective inhibition of protein kinase $\mathrm{C}$ beta2 attenuates the adaptor P66 Shcmediated intestinal ischemia-reperfusion injury. Cell Death Dis. (2014) 5:e1164. doi: 10.1038/cddis.2014.131

64. Zhao Y, Wang Z, Feng D, Zhao H, Lin M, Hu Y, et al. p66Shc contributes to liver fibrosis through the regulation of mitochondrial reactive oxygen species. Theranostics. (2019) 9:1510-22. doi: 10.7150/thno.29620

65. Sampaio SF, Branco AF, Wojtala A, Vega-Naredo I, Wieckowski MR, Oliveira PJ. p66Shc signaling is involved in stress responses elicited by anthracycline treatment of rat cardiomyoblasts. Arch Toxicol. (2016) 90:166984. doi: 10.1007/s00204-015-1583-9

66. Wang G, Yao J, Li Z, Zu G, Feng D, Shan W, et al. miR-34a-5p inhibition alleviates intestinal ischemia/reperfusion-induced reactive oxygen species accumulation and apoptosis via activation of SIRT1 signaling. Antioxid Redox Signal. (2016) 24:961-73. doi: 10.1089/ars.2015.6492

67. Wu B, Ootani A, Iwakiri R, Fujise $T$, Tsunada $S$, Toda $S$, et al. Ischemic preconditioning attenuates ischemia-reperfusion-induced mucosal apoptosis by inhibiting the mitochondria-dependent pathway in rat small intestine. Am J Physiol Gastrointest Liver Physiol. (2004) 286:G5807. doi: 10.1152/ajpgi.00335.2003

68. Wang G, Chen Z, Zhang F, Jing H, Xu W, Ning S, et al. Blockade of PKCbeta protects against remote organ injury induced by intestinal ischemia and reperfusion via a p66shc-mediated mitochondrial apoptotic pathway. Apoptosis. (2014) 19:1342-53. doi: 10.1007/s10495-014-1008-x

69. Gebert LFR, MacRae IJ. Regulation of microRNA function in animals. Nat Rev Mol Cell Biol. (2019) 20:21-37. doi: 10.1038/s41580-018-0045-7

70. Akbari G. Emerging roles of microRNAs in intestinal ischemia/reperfusioninduced injury: a review. J Physiol Biochem. (2020) 76:52537. doi: 10.1007/s13105-020-00772-y

71. Li LX, Yin LH, Gao M, Xu LN, Qi Y, Peng JY. MiR-23a-5p exacerbates intestinal ischemia-reperfusion injury by promoting oxidative stress via targeting PPAR alpha. Biochem Pharmacol. (2020) 180:114194. doi: 10.1016/j.bcp.2020.114194

72. Hu Y, Tao X, Han X, Xu L, Yin L, Sun H, et al. MicroRNA-351-5p aggravates intestinal ischaemia/reperfusion injury through the targeting of MAPK13 and sirtuin-6. Br J Pharmacol. (2018) 175:3594-609. doi: 10.1111/bph.14428

73. Chen $\mathrm{C}$, Zhou M, Ge Y, Wang X. SIRT1 and aging related signaling pathways. Mech Ageing Dev. (2020) 187:111215. doi: 10.1016/j.mad.2020.111215

74. Kong X, Guan J, Li J, Wei J, Wang R. P66(Shc)-SIRT1 regulation of oxidative stress protects against cardio-cerebral vascular disease. Mol Neurobiol. (2017) 54:5277-85. doi: 10.1007/s12035-016-0073-2

75. Chai D, Zhang L, Xi S, Cheng Y, Jiang H, Hu R. Nrf2 activation induced by sirtl ameliorates acute lung injury after intestinal ischemia/reperfusion through NOX4-mediated gene regulation. Cell Physiol Biochem. (2018) 46:781-92. doi: 10.1159/000488736

76. Zheng L, Han X, Hu Y, Zhao X, Yin L, Xu L, et al. Dioscin ameliorates intestinal ischemia/reperfusion injury via adjusting miR-351-5p/MAPK13mediated inflammation and apoptosis. Pharmacol Res. (2019) 139:4319. doi: 10.1016/j.phrs.2018.11.040

77. Lerrer B, Gertler AA, Cohen HY. The complex role of SIRT6 in carcinogenesis. Carcinogenesis. (2016) 37:108-18. doi: 10.1093/carcin/bgv167

78. Ran LK, Chen Y, Zhang ZZ, Tao NN, Ren JH, Zhou L, et al. SIRT6 overexpression potentiates apoptosis evasion in hepatocellular carcinoma via BCL2-associated X protein-dependent apoptotic pathway. Clin Cancer Res. (2016) 22:3372-82. doi: 10.1158/1078-0432.CCR-15-1638

79. Bock FJ, Tait SWG. Mitochondria as multifaceted regulators of cell death. Nat Rev Mol Cell Biol. (2020) 21:85-100. doi: 10.1038/s41580-019-0173-8

80. West AP, Shadel GS. Mitochondrial DNA in innate immune responses and inflammatory pathology. Nat Rev Immunol. (2017) 17:363-75. doi: 10.1038/nri.2017.21

81. Islam MT. Oxidative stress and mitochondrial dysfunctionlinked neurodegenerative disorders. Neurol Res. (2017) 39:7382. doi: 10.1080/01616412.2016.1251711

82. Yu EP, Bennett MR. The role of mitochondrial DNA damage in the development of atherosclerosis. Free Radic Biol 
Med. (2016) 100:223-30. doi: 10.1016/j.freeradbiomed 2016.0 6.011

83. Liu S, Feng M, Guan W. Mitochondrial DNA sensing by STING signaling participates in inflammation, cancer and beyond. Int $J$ Cancer. (2016) 139:736-41. doi: 10.1002/ijc.3 0074

84. Hu Q, Ren J, Wu J, Li G, Wu X, Liu S, et al. Elevated levels of plasma mitochondrial DNA are associated with clinical outcome in intra-abdominal infections caused by severe trauma. Surg Infect. (2017) 18:610-8. doi: 10.1089/sur.201 6.276

85. Fang C, Wei X, Wei Y. Mitochondrial DNA in the regulation of innate immune responses. Protein Cell. (2016) 7:11-6. doi: 10.1007/s13238-015-0 222-9

86. Hu Q, Ren J, Li G, Wu J, Wu X, Wang G, et al. The mitochondrially targeted antioxidant MitoQ protects the intestinal barrier by ameliorating mitochondrial DNA damage via the Nrf2/ARE signaling pathway. Cell Death Dis. (2018) 9:403. doi: 10.1038/s41419-018-0436-x
Conflict of Interest: The authors declare that the research was conducted in the absence of any commercial or financial relationships that could be construed as a potential conflict of interest.

Publisher's Note: All claims expressed in this article are solely those of the authors and do not necessarily represent those of their affiliated organizations, or those of the publisher, the editors and the reviewers. Any product that may be evaluated in this article, or claim that may be made by its manufacturer, is not guaranteed or endorsed by the publisher.

Copyright (c) $2022 \mathrm{Li}$, Wang and Fan. This is an open-access article distributed under the terms of the Creative Commons Attribution License (CC BY). The use, distribution or reproduction in other forums is permitted, provided the original author(s) and the copyright owner(s) are credited and that the original publication in this journal is cited, in accordance with accepted academic practice. No use, distribution or reproduction is permitted which does not comply with these terms. 